\title{
Surgical Challenges of Heart Mate 3 Pump Implantation in Aneurysmally Changed Heart Ventricles
}

\author{
Dusko Terzić, ${ }^{1,2}$ Emilija Nestorović, ${ }^{1,2}$ Dejan Markovic, ${ }^{1,2,3}$ Nataša Kovačević Kostić, ${ }^{1,3}$ Aleksandar Djordjevic, ${ }^{1,2}$ \\ Radmila Karan, ${ }^{1,2,3}$ Marko Čubrilo, ${ }^{1,2}$ Vladimir Stoiljković, ${ }^{4}$ Vladimir Milićević, ${ }^{1}$ \\ Jelena Cumic ${ }^{1,3}$ Svetozar Putnik,,$^{1,2}$ \\ ${ }^{1}$ Department for Heart Transplant, LVAD and ECMO, Clinic for Cardiac surgery, Clinical Center of Serbia, Belgrade, Serbia; \\ ${ }^{2}$ School of Medicine, University of Belgrade, Serbia; ${ }^{3}$ Clinical Center of Serbia, Center for Anesthesiology and Reanimation, \\ Belgrade, Serbia; ${ }^{4}$ Clinical Center of Niš, Serbia
}

\section{ABSTRACT}

We presented surgical treatment of three patients indicated for implantation of a permanent mechanical circulatory support device and with the associated left ventricular aneurysms. In order to evade the left ventricular rupture, adverse thromboembolic events and provide safe implantation of the inflow c annula, L VAD H M3 i mplantation together with the reconstruction of the left ventricular aneurysmal wall was performed in two patients. Regarding the third patient, LVAD implantation upon the reconstruction of the left ventricular wall was abandoned because there was no safe location for placement of the inflow cannula.

\section{INTRODUCTION}

Heart transplantation is the method of choice in treatment of end stage heart failure. Given the enormous issue of an insufficient number of donors as well as a considerable number of contraindications for surgery, particularly among the older population, there is a strong need for alternative strategy; therefore, the role of the mechanical circulatory support device has been more pronounced, either it is applied as a bridge to transplantation or destination therapy [Netuka 2015; Patel 2016].

The basic problem of LVAD implantation in patients with the left ventricular aneurysm is a fixation of the inflow cannula to thinned, cicatricially-changed myocardium of the left ventricle. Moreover, altered endocardium of the respective segment may be a significant source of thrombotic masses in conditions of nonpulsatile flow, resulting in thromboembolic complications and pump thrombosis. Finally, inadequately fixed inflow cannula may lead to suction of the left ventricular

Received Auguts 25, 2019; received in revised form fanuary 2, 2020; accepted fanuary 18, 2020.

Correspondence: Dusko Terzić, Department for Heart Transplant, LVAD and ECMO, Clinic for Cardiac surgery, Clinical Center of Serbia, Belgrade, Serbia (e-mail: terzic.dusko@gmail.com). myocardium and mitral valve device components with potentially disastrous complications.

Patient 1: A 56-year-old male, with severe general condition, was diagnosed with dilated ischemic cardiomyopathy. It was categorized as NYHA class IV, INTERMACS class 3. The patient had the acute anterior wall myocardial infarction seven months before admission to our institution.

Echocardiography revealed extremely dilated left ventricle with EDD $7.3 \mathrm{~cm}$, ESD $6.8 \mathrm{~cm}$, and modified apical segment in the form of huge pseudoaneurysm of $9.3 \times 9.4 \mathrm{~cm}$ in size, which involved the apex, and the apical and medial segments of all left ventricular walls without any apparent thrombotic mass. LV systolic function significantly was diminished (EF

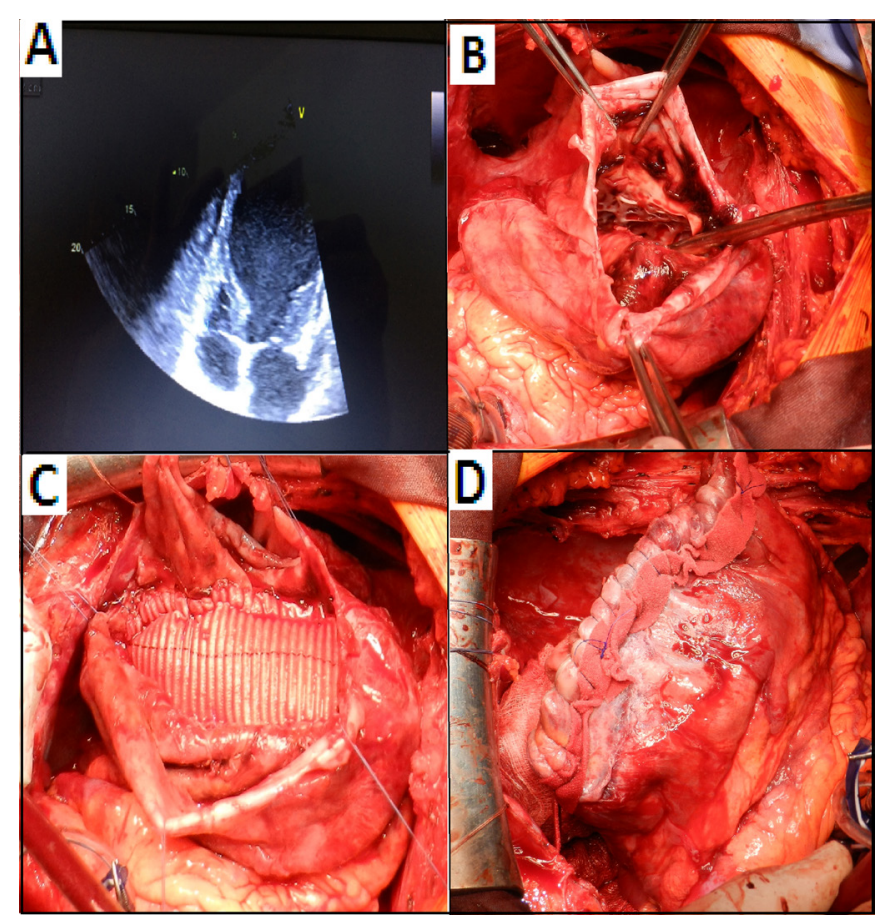

Figure 1. A, Echocardiography -apical segment in the form of huge pseudoaneurysm of $9.3 \times 9.4 \mathrm{~cm}$; B, resected aneurysm; C, left ventricular wall reconstructed by dacron; $\mathrm{D}$, dacron patch covered by resected myocardial tissue. 


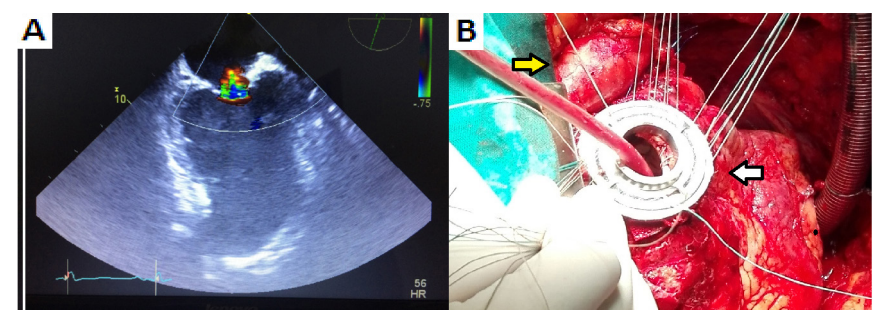

Figure 2. A, Echocardiography - apical aneurysm $5.4 \times 4.2 \mathrm{~cm}$; B, Aneurysmal neck was plicated with teflon felt, enabling the separation of aneurysmal cavity from the left ventricular residue (yellow arrow). Surgical creation of an opening in the ventricle above the location of the aneurysmal plication (white arrow).

by Simpson method 10\%, EF by Teicholz method 12\%). The left atrium was enlarged, being $5.0 \mathrm{~cm}$ in size. The dimensions of the right ventricle were regular, and its longitudinal and systolic functions were normal, TAPSE $1.4 \mathrm{~cm}$ with trace of TR+. Coronarographic examina-tion showed the LAD coronary artery occlusion.

Intraoperatively, a huge pseudoaneurysm of the left ventricle sized $12 \times 10 \mathrm{~cm}$ was evident, as sequelae of the rupture of the left ventricular free walls accompanied with massive pericardial adhesions. The aneurysm was resected and the left ventricular wall was reconstructed by dacron patch, while suture structures were covered by resected myocardial tissue (Figure 1). It was confirmed that there was no sufficiently large area of the adequate myocardial tissue for safe positioning of LVAD inflow cannula. Considering the patient's hemodynamic stabilization with inotropic support and echocardiographically verified improvement of the left ventricular function, it was concluded to abandon, in this phase, the idea of implanting the mechanical circulatory support system. The patient successfully was separated from the extracorporeal circulation system. Early and late postoperative periods were uneventful. The patient was discharged after 30 days. After six months, he underwent successful heart transplantation.

Patient 2: A 59-year-old male in end stage heart failure, with experienced anterior myocardial infarction 14 years ago and resulting left ventricular aneurysm, presented to our institution. The patient also had Neurofibromatosis Recklinghausen (nonmalignant) and hypothyreosis. It was categorized as NYHA class IV, INTERMACS class 3. Coronarography revealed LAD occlusion with subocclusion of D1 and $\mathrm{Cx}$ branches. On admission, echocardiographic find-ing showed a dilated left ventricle $(8,2 / 7,0 \mathrm{~cm})$, with apical aneu$\operatorname{rysm}(5.4 \times 4.2 \mathrm{~cm})$, and EF (15\% by Simpson method, $22 \%$ by Teicholz method). MR 2-3+ and enlarged left atrium, 6.7 $\mathrm{cm}$ in size, were recorded. The right ventricle had normal dimensions $(2.8 \mathrm{~cm})$, and good sys-tolic and longitudinal functions, TAPSE $22 \mathrm{~mm}$. In addition, the apical aneurysm of $6 \times 6.5 \mathrm{~cm}$ in size, impregnated with calcium deposits, also was verified. It was estimated that the left ventricular apex, given its brittleness and the lack of adequate support, was not suitable for placement of LVAD inflow cannula. Aneurysmal neck was plicated with teflon felt, enabling the separation of the aneurysmal cavity from the left ventricular residue. After

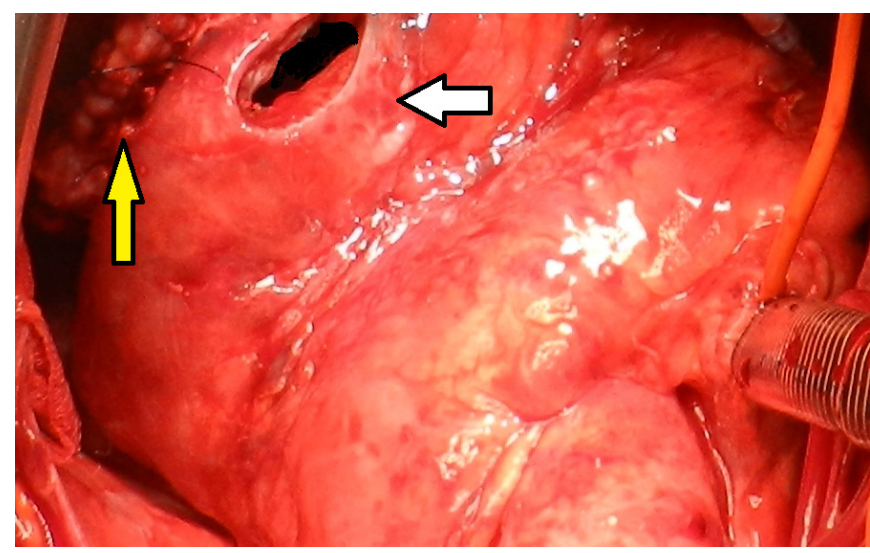

Figure 3. The latero-diaphragmal part of apex was plicated (yellow arrow) and the entire ventricular wall (full thickness) was sutured (white arrow).

the surgical creation of an opening in the ventricle above the location of the aneurysmal plication, fixation ring successfully was sutured and LVAD was implanted (Figure 2). Considering the large dimension of the ventricle itself, there were no hemodynamic effects on pump function, which was implanted laterally and above the ventricular apex, presenting an unusual position for such procedure. The patient was transferred to the intensive care unit hemodynamically stable with pump parameters pf $3.21 / \mathrm{min}$, ps $5000 \mathrm{rpm}$, pi 4.8 , and pp $3.2 \mathrm{w}$. The patient de-veloped postoperative pneumonia and Acinetobacter sp. was cultured from sputum. The expected outcome was not achieved with antibiotic treatment. Two months after the implanta-tion, the patient died from pneumonia-related complications. During that period, myocardial pump parameters were regular.

Patient 3: The patient was a 54-year-old male in end stage heart failure. Ten months before his hospitalization, he had an acute myocardial infarction of the anterior wall as the first manifestation of the coronary disease. After the acute ischemic phase, hospital course was complicated by development of the left ventricular aneurysm and presentation of heart failure symptoms. Coronarographic finding revealed the LAD coronary artery occlusion as well as the stenosis of the proximal circumflex coronary and right coronary arteries.

Cardiac echocardiography showed aorta with normal bulbal width $(3.6 \mathrm{~cm})$. The left ventricle had bigger dimensions (EDD $7.8 \mathrm{~cm}$, ESD $6.7 \mathrm{~cm}$ ) and reduced systolic function by Simpson method, i.e., EF 18\%; in addition, the left ventricle with the akinetic septum, apex, and ante-rior wall, had poor function. An apical aneurysm $5.2 \times 4.4 \mathrm{~cm}$ in size was evident, but with-out any apparent thrombotic mural formations. Moderate to severe MR 2-3+ was recorded. The right ventricle had normal dimensions $(2.6 \mathrm{~cm})$ and normal systolic and longitudinal functions; tricuspid valve function was preserved.

SPECT ventriculography demonstrated the absence of viable myocardium of the anterior and lateral walls of the left ventricle.

The condition was categorized as NYHA class IV, INTERMACS class 3 as sequelae of the severe ischemic cardiomyopathy. 
LVAD HM III was implanted as BTT. Due to aneurysmic dilatation of the left ventricular apex and for proper and safe fixation of the ring, the latero-diaphragmal part of apex was pli-cated and the left ventricle was opened at its apical part, and the entire ventricular wall (full thickness) was sutured (in distinction from regular practice of suturing, which does not pass through full ventricular wall thickness before opening the ventricle) (Figure 3). The sutures were strengthened by teflon felt. The surgical intervention was uneventful. Pump speed was set to 4900rpm, PF 3.4, PP 3.2 and PI 3.3. Postoperative course was uneventful, and the pa-tient was discharged two weeks later. The patient has been on the transplant waiting list at one year, during his follow-up period.

\section{DISCUSSION}

Left ventricular aneurysms are defined as limited areas of abnormal diastolic contour of the left ventricle with systolic dyskinesia and paradoxical inflation. The left ventricular aneurysms basically are divided to true aneurysms and pseudo aneurysms. The wall of true aneurysm consists of endocardium, epicardium and thinned cicatricial tissue located on earlier myocardium. From functional aspect, true aneurysm may be dyskinetic and akinetic. Pseudoaneurysm is the result of the left ventricle rupture, whereby its walls are consisted of peri-cardium tissue, connective tissue of pericardial adhesions, thrombotic masses, and probably epicardium.

Implantation of devices for mechanical circulation support has nowadays become a routine procedure, which is carried out in numerous surgical institutions with minimal complications. One of the most significant problems, during LVAD implantation, is adequate positioning of the inflow cannula. The position of the inflow cannula is of crucial importance for adequate LVAD function. It is recommended that the inflow cannula should be aligned with the interventricular septum and placed directly against the mitral valve [Zimpfer 2016].

Inadequate cannula position may result in poor inflow, myocardial suction (often suction events), turbulent blood flow (with subsequent hemolysis and thrombosis), all together lead-ing to potentially disastrous complications. This problem particularly is pronounced in pa-tients with simultaneously present aneurysm of the left ventricle or those who have previously undergone left ventricle reconstruction in the zone of the aneurysm.

Earlier approach in these patients involved the implantation of extracorporeal devices for mechanical circulation support, along with placement of the inflow cannula in the left atrium. Today, this approach is considered inadequate, before all in the group of patients in whom LVAD presents definitive therapy [Garbade 2011].

Simultaneous left ventricular reconstruction, which changes the geometry and achieves more regular elliptical shape of the ventricle, ensures the safe location for placement of the inflow cannula as well as its adequate orientation. In addition, alternative sites for inflow cannula placement, such as beyond the ventricular apex, have been described in reports.
Garbade and associates present Dor LVAD implantation procedure (ThoratecHeartMate II; Thoratec Corporation, Pleasanton, CA) with inflow cannula fixation to the synthetic patch. Similar technique was described by Chernyavski and associates (Berlin Heart INCOR; Berlin Heart Inc., The Woodlands, TX) who implanted LVAD in the same way. The limitation of this technique is instability of cannula as well as achievement of adequate hemostasis [Gar-bade 2011; Palmen 2012].

Available articles describe LVAD implantation to preserved apical part, without any synthetic material applied, but with prior partial reconstruction of the left ventricle [Fatullayev 2014; Chernyavskiy 2012].

Williams et al applies the method involving the inferiorlateral insertion of inflow cannula in relation to the position of the former apex, which is limited by ventriculostomy scar. The patch, used for reconstruction of the true apex, is left in place and serves as orientation for inflow cannula insertion. The authors report that this technique is suitable for LVAD implantation, when it is placed in the abdominal pocket, while it may be quite inadequate if placing LVAD to a completely pericardial position [Williams 2006].

Palmen et al describe the technique, including the total ventricular scar excision with reconstruction of the neo apex around the Hegar dilator, which is applied for pinpointing the optimal position and orientation of the inflow cannula [Palmen 2012].

All three patients had an indication for LVAD implantation. In our opinion, reconstruction of the left ventricular aneurysm, as part of LVAD implantation, may be an ancillary method (case of the second patient) or an involuntary method (case of the first patient). The most important factors associated with the decision to abandon LVAD implantation are residual tissue quality in the inflow cannula insertion zone, absence of calcium deposits, and myocardial functional reserve potential.

Regarding the first patient, it was not possible to insert inflow cannula either because of the lack of adequate tissue or impossibility to find a suitable alternative to the insertion site, and, therefore, it was decided to abandon the LVAD intervention. The patient possessed sufficient cardiac functional reserve to live until heart transplantation, given that the ventricular reconstruction produced better function, especially of basal segments.

Considering the second patient, the cannula was placed to an alternative position above and lateral to the ventricular apex, and in postoperative course, the position of cannula had no negative effect on pump function. Poor clinical outcome after two months, due to respiratory complications, cannot be associated with cannula positioning.

The third patient had the least manifested apical aneurysm and no calcium deposits. Accordingly, the inflow cannula insertion through the aneurysm was possible. The technique was modified in such a way that earlier surgical creation of the circular opening of ventricle enabled the pledgeted sutures to run through the full thickness of the left ventricular wall (given that common technique of HM 3 pump implantation assumes the placement of ring fixation sutures before opening the ventricle). 


\section{CONCLUSION}

LVAD implantation could be a mode of treatment opted for select patients with large aneurysms of the left ventricle, considering noticeable surgical risks due to inadequate tissue consistency of the left ventricle and thromboembolic characteristics of the aneurysmal tissue.

\section{REFERENCES}

Chernyavskiy AM, Marchenko AV, Lomivorotov VV, Doronin D, Alsov SA, Nesmachnyy A. 2012. Left ventricular assist device implantation combined with surgical ventricular re-construction. Tex Heart Inst J. 39(5): 627-629.

Fatullayev J, Butters T, Sabashnikov A, et al. 2014. Left ventricular assist device implanta-tion with concomitant left ventricular reconstruction without patchplasty. J Artif Organs. 17(4):370-372.

Garbade J, Bittner HB, Barten MJ, et al. 2011. Combined surgical left ventricular reconstruc-tion and left ventricular assist device implantation for destination therapy in end-stage heart failure. Circ Heart Fail. 4:e14-e15.

Netuka I, Sood P, Pya Y, et al. 2015. Fully Magnetically Levitated Left Ventricular Assist System for Treating Advanced HF: A Multicenter Study. J Am Coll Cardiol 66:2579-89.

Palmen M, Verwey HF, Haeck ML, et al. 2012. Implantation of a left ventricular assist de-vice in patients with a complex apical anatomy. Ann Thorac Surg 94:2122-5.

Patel S, Nicholson L, Cassidy CJ, Wong KY. 2016. Left ventricular assist device: A bridge to transplant or destination therapy? Postgrad Med J. 92:271-81.

Williams J, Conte J. 2006. Ventricular assist device placement following surgical ventricular restoration. Interact Cardiovasc Thorac Surg 5:90 -1 .

Zimpfer D, Netuka I, Schmitto JD, Pya Y, Garbade J, Morshuis et al. 2016. Multicentre clini-cal trial experience with the HeartMate 3 left ventricular assist device: 30-day outcomes. Eur J Cardiothorac Surg Sep;50(3):548-54. 